\title{
MEDICATION DISPENSING ERRORS IN URBAN PHARMACIES: IMPLEMENTING BARCODE TECHNOLOGY
}

\author{
Mohsen Masoumi ${ }^{1}$, Mohammad Reza Hasibian ${ }^{2}$, Hasan Vakili-Arki ${ }^{1}$, Zhila Taherzadeh ${ }^{3}$, Ehsan Nabovati ${ }^{4}$,
} Naser Vahdati Mashhadian ${ }^{5}$, Saeid Eslami ${ }^{2,6,7, *}$

1: Student Research Committee, Department of Medical Informatics, Faculty of Medicine, Mashhad University of Medical Sciences, Mashhad, Iran

2: Department of Medical Informatics, Faculty of Medicine, Mashhad University of Medical Sciences, Mashhad, Iran

3: Targeted Drug Delivery Research Center and Neurogenic Inflammation Research Center, School of PharmacyMashhad, University of Medical Sciences, Mashhad, Iran

4: Health Information Management Research Center, Kashan University of Medical Sciences, Kashan, Iran

5: Toxicology Research Center, Mashhad University of Medical Sciences, Mashhad, Iran

6: Pharmaceutical Research Center, School of Pharmacy, Mashhad University of Medical Sciences, Mashhad, Iran

7: Department of Medical Informatics, Academic Medical Center, Amsterdam, The Netherlands

Correspondence:

Saeed Eslami. Pharmaceutical Research Center, School of Pharmacy, Mashhad University of Medical Sciences, Mashhad, Iran; Department of Medical Informatics, School of Medicine, Mashhad University of Medical Sciences, Mashhad, Iran; Department of Medical Informatics, Academic Medical Center, Amsterdam, The Netherlands

Tel: +98.51-380022429, Fax: +98.51-38827048, E-mail: EslamiS@mums.ac.ir

\section{TYPE OF ARTICLE: CONFERENCE ABSTRACT}

\begin{abstract}
Introduction: The importance of patient safety has drawn attention to reducing and preventing medication errors, which can lead to increased effort in using information technology systems. Using barcode technology in the pharmacy could reduce dispensing errors, but data about its effect are limited. The aim of this study was to evaluate the frequency of medication dispensing errors in pharmacies, which are equipped/nonequipped with barcode scanning systems.

Methods: This research was a prospective and quasi-experimental "post-intervention with equivalent control group" study carried out in two community pharmacies in Mashhad, Iran, from January to February 2015. In a pharmacy, a barcode scanning system was used for recognizing medication (Pharmacy A), while such a system was not used in the other one (Pharmacy B). The study population consisted of all the received prescriptions in both pharmacies during a period of two months. The number of relevant transcription, prescription filling and pharmacist action errors (wrong strength, wrong dispensing form, wrong medicine, wrong quantity, omission errors) were assessed. The traveled distance for preparing prescriptions and patient waiting time also were considered as outcomes.

Results: A total of 2386 and 900 paper prescriptions were received by the Pharmacy A and Pharmacy B, respectively. There were 211 (7/7\%) dispensing errors in Pharmacy A and $113(10 \%)$ dispensing errors in the Pharmacy B $(p<0 / 05)$. In Pharmacy A, most error or deficiencies in all prescriptions were found in quantity (4.44\%) followed by medicine $(2.26 \%)$, strength $(1.09 \%)$, omission $(0.67 \%)$, and dispensing form $(0.38 \%)$. In Pharmacy B, the frequencies of the errors in all prescriptions vary in the same order, ranging from $5.78 \%$ for the wrong quantity, to $4.89 \%$ for the wrong medicine, to $1.78 \%$ for wrong strength, and to $0.11 \%$ for the wrong dispensing form. No case with omission error was found in Pharmacy B. There were statistical significant differences between the two pharmacies in terms of "wrong medicine" and "omission error" $(p<0.05)$. The percentage of transcription, prescription filling, and pharmacist action errors in proportion of all prescriptions of Pharmacy A were calculated at 3.81\%, 4.86\%, and $0.17 \%$ and in Pharmacy B $6.67 \%, 5.56 \%$, and $0.33 \%$,
\end{abstract}

\section{Abstracts of First National Congress of Medical Informatics, Mashhad, Iran, February 2017}

(C) 2017 The Authors. This is an open access article under the terms of the Creative Commons Attribution-NonCommercialNoDerivs License, which permits use and distribution in any medium, provided the original work is properly cited, the use is non-commercial and no modifications or adaptations are made. 
respectively ( $p=0.005, p=0.418, p=0.385$, respectively). The error rates related to medicine were detected at $12.9 \%$ in Pharmacy A and 30\% in Pharmacy B $(p=0.013)$. The traveled distance per each medicine item during the prescription-filling process was 4.4 and $1.5 \mathrm{~m}$ in Pharmacy $\mathrm{A}$ and $\mathrm{B}$, respectively.

Conclusion: Using a barcode scanning system in a pharmacy did not remove all medication errors, but it was associated with reduction of dispensing errors and could improve patient safety.

KEYWORDS: Barcode scanning system, Dispensing errors, Pharmacy, Patient safety 